\title{
Three-Dimensional Representation of Complex Muscle Architectures and Geometries
}

\author{
SiLVIA S. BlemKER and SCOTT L. DelP \\ Departments of Bioengineering and Mechanical Engineering, Stanford University, Stanford, CA
}

(Received 1 July 2004; accepted 17 November 2004)

\begin{abstract}
Almost all computer models of the musculoskeletal system represent muscle geometry using a series of line segments. This simplification (i) limits the ability of models to accurately represent the paths of muscles with complex geometry and (ii) assumes that moment arms are equivalent for all fibers within a muscle (or muscle compartment). The goal of this work was to develop and evaluate a new method for creating three-dimensional (3D) finite-element models that represent complex muscle geometry and the variation in moment arms across fibers within a muscle. We created 3D models of the psoas, iliacus, gluteus maximus, and gluteus medius muscles from magnetic resonance (MR) images. Peak fiber moment arms varied substantially among fibers within each muscle (e.g., for the psoas the peak fiber hip flexion moment arms varied from 2 to $3 \mathrm{~cm}$, and for the gluteus maximus the peak fiber hip extension moment arms varied from 1 to $7 \mathrm{~cm}$ ). Moment arms from the literature were generally within the range of fiber moment arms predicted by the $3 \mathrm{D}$ models. The models accurately predicted changes in muscle surface geometry over a $55^{\circ}$ range of hip flexion, as compared to changes in shape predicted from MR images (average errors between the model and measured surfaces were between 1.7 and $5.2 \mathrm{~mm}$ ). This new framework for representing muscle will enhance the accuracy of computer models of the musculoskeletal system.
\end{abstract}

Keywords-Skeletal muscle, Finite-element modeling, Musculoskeletal geometry, Moment arms, Lower limb.

\section{INTRODUCTION}

Accurate descriptions of muscle geometry are needed to characterize muscle function. The length and moment arm of a muscle affect the muscle's ability to generate force, produce joint moments, and actuate movement. However, the geometrical arrangement of skeletal muscles is complex. Each joint is spanned by several muscles that are closely packed together and have a variety of shapes and sizes. As joints move, muscles change shape and interact with each other and with underlying bones. Computer models of the musculoskeletal system generally simplify muscles by representing them as a series of line segments ${ }^{12,16,27}$

Address correspondence to Scott L. Delp, James H. Clark Center, Room S-321, Stanford University, MailCode 5450, 318 Campus Drive, Stanford, CA 94305-5450. Electronic mail: delp@stanford.edu that pass through the approximate centroidal path of the muscle and represent the muscle's effective line of action. ${ }^{29}$

Using a series of line segments to represent the path of a muscle with complex geometry is difficult because it requires knowledge of how the muscle changes shape and interacts with underlying muscles, bones, and other structures as joints move. For example, it is challenging to represent muscles that wrap and bend around underlying structures, like the psoas muscle (Fig. 1(A)). "Via points" and "wrapping surfaces" are commonly defined to geometrically constrain the path from penetrating underlying bones and muscles ${ }^{15,22,45}$; however, it is frequently unclear how to specify these constraints, and the muscle moment arms can be highly sensitive to how the constraints are defined. It also is challenging to use a series of line segments to represent muscles with broad attachments, like the gluteus maximus (Fig. 1(B)). Models generally separate these types of muscles into compartments and use multiple paths to represent the muscle. ${ }^{44}$ However, it is often unclear how many paths to define, where the paths should be located, and how to define via points (and/or wrapping surfaces) so that the models accurately represent the anatomy.

Modeling muscle using a series of line segments allows only one length and moment arm to be estimated for each muscle path. Therefore, "lumped-parameter" models, ${ }^{49}$ which assume that all fibers within a muscle have the same length and moment arm, are used to estimate muscle force. However, variation in moment arms (and therefore changes in length) among fibers within a muscle could greatly influence the muscle's capacity to generate force. Indeed, previous studies (e.g., Herzog and ter Keurs ${ }^{26}$ ) have demonstrated that lumped-parameter models do not accurately predict in vivo force-joint angle behaviors, especially for muscles with complex geometries and architectures. Models that represent the three-dimensional (3D) arrangement of muscle fibers and allow for variations in fiber lengths and moment arms are needed to more closely represent in vivo muscle behavior.

The goal of this work was to create and evaluate a new representation for muscle that can characterize a range of 


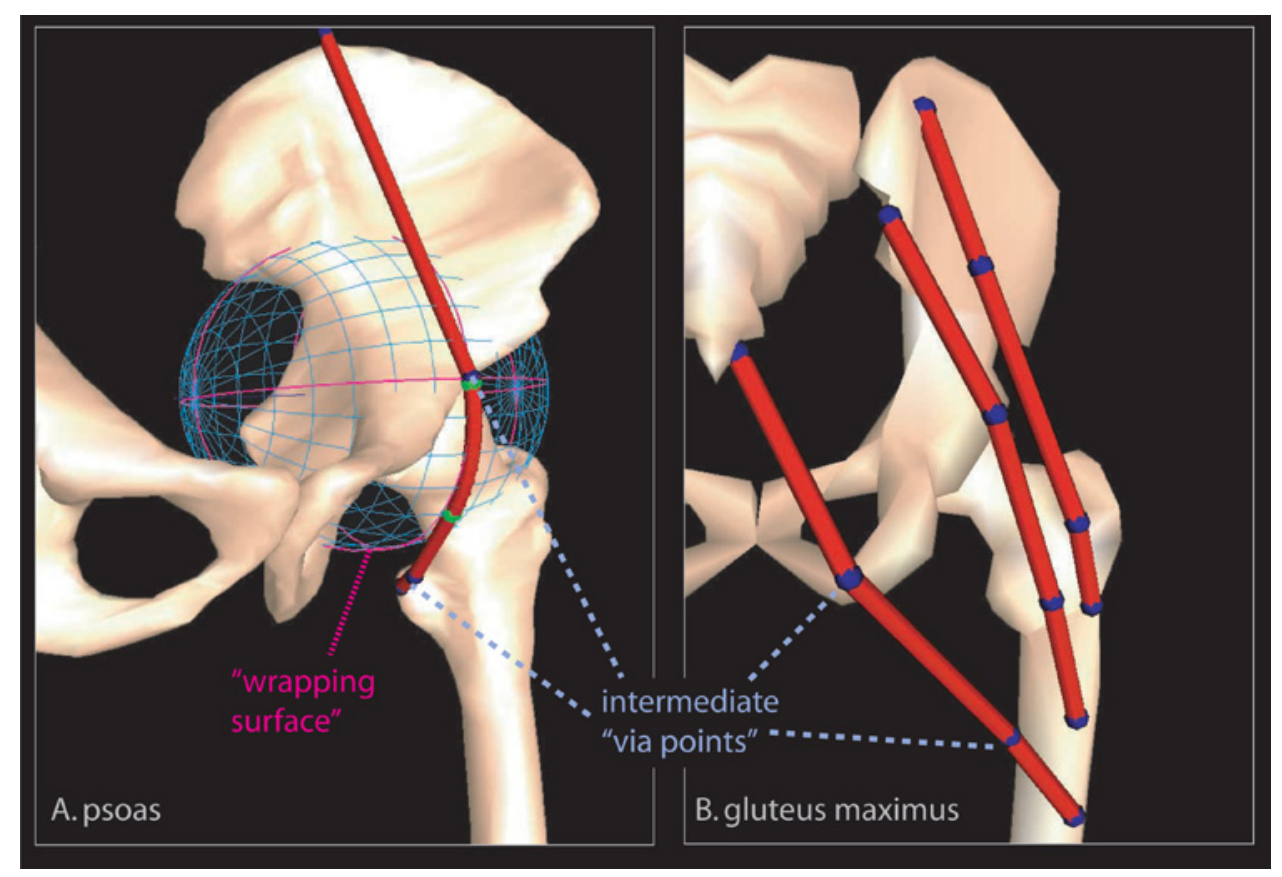

FIGURE 1. Series-of-line-segments simplification generally used in musculoskeletal modeling., ${ }^{6,16}$ The psoas muscle (A) has a complex path that bends, or wraps, around the pelvic brim, hip joint capsule, and the femoral neck. With the 1D techniques, "via points" (blue) and "wrapping surfaces" (wireframe) are used to prevent the muscle from penetrating these structures. However, it is difficult to define via points and wrapping surfaces that work robustly for multiple degrees of freedom. The gluteus maximus muscle $(B)$ is also difficult to model because it has broad areas of attachment and the fibers have a complex geometric arrangement.

fiber lengths and moment arms in muscles with complex geometry. This new representation addresses two major limitations of models that characterize muscles with a series of line segments: (i) their limited capacity for representing muscles with complex geometry and (ii) their assumption that all fibers within each muscle compartment have the same length and moment arm. The representations incorporate the $3 \mathrm{D}$ geometry of the muscles, the $3 \mathrm{D}$ arrangement of the muscle fibers, the constitutive properties of muscle and tendon, and the mechanics of the interactions with neighboring structures. We evaluated the utility and accuracy of this method by creating models of four muscles that cross the hip (gluteus maximus, gluteus medius, psoas, and iliacus). These muscles wrap around underlying structures, have broad attachments, and have a variety of architectures. The 3D models of these muscles highlight the diverse behaviors among the fibers within muscles and illustrate the value of this new approach.
A.

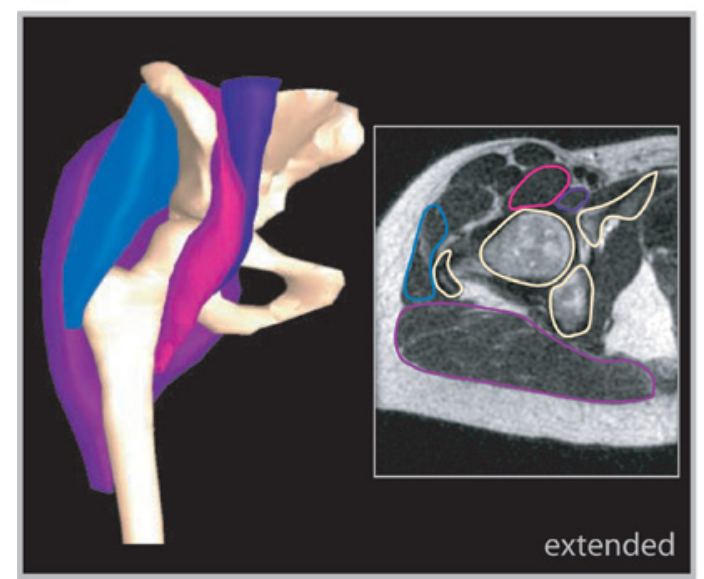

B.

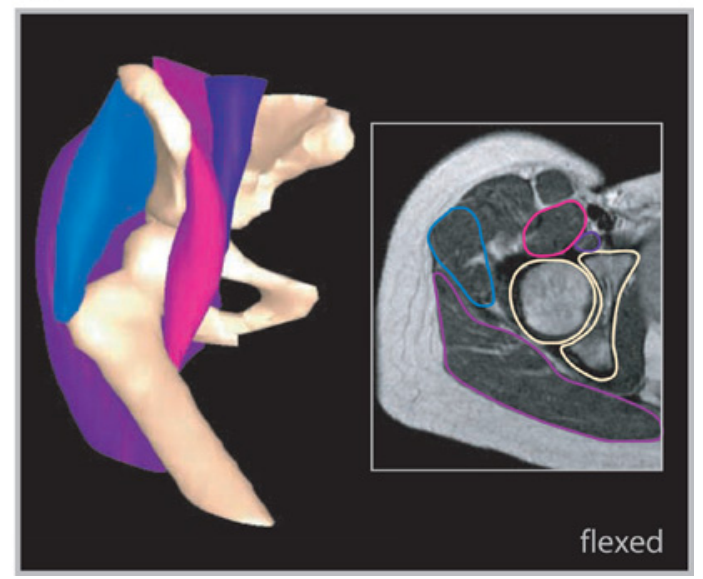

FIGURE 2. Segmentation of MR images to create surface models. In the extended position (A) and flexed (B) positions, the gluteus maximus, gluteus medius, iliacus, psoas, pelvis, and femur were outlined in each axial image, and surface models were created. 


\section{METHODS}

We created 3D finite-element models of the muscles of interest and their underlying bones from magnetic resonance (MR) images of a live subject. Finite-element meshes and geometric descriptions of the fibers were created for each muscle. A transversely-isotropic, quasiincompressible, hyperelastic constitutive model was used to represent the stress-strain behavior of muscle and tendon. The origin and insertion regions of each muscle mesh were defined to rigidly attach to their corresponding bones, and finite-element simulations through ranges of hip flexionextension, adduction-abduction, and internal-external rotation were performed. Fibers were tracked throughout the simulations, and ranges of fiber lengths and moment arms were calculated for each muscle. We evaluated the models by (i) comparing the predicted fiber moment arms with muscle moment arms determined from previous experimental measurements and models from the literature, and (ii) comparing the change in shape predicted by models with 3D surfaces measured from MR images.

\section{Construction of Finite-Element Meshes from Image Data}

The goal of the imaging session was to capture the detailed hip and thigh anatomy at approximately 0 and $60^{\circ}$ of hip flexion. The subject (female; height $160 \mathrm{~cm}$; age 27 years) was positioned supine on the table of a 1.5-T GE scanner (GE Medical Systems, Milwaukee, Wiscon$\sin )$. The lower limb was first situated with the hip extended (approximately $0^{\circ}$ of hip flexion) and the knee extended. In this position, we acquired two sets of proton-density (repetition time $=4000 \mathrm{~ms}$; echo time $=11.3 \mathrm{~ms}$; echo train length $=8)$ axial images. The first series $(30 \mathrm{~cm} \times$ $30 \mathrm{~cm}$ field of view; $4 \mathrm{~mm}$ slice thickness, $1 \mathrm{~mm}$ space between slices; 256 pixel $\times 256$ pixel matrix) traveled from just below the iliac crest to just below the lesser trochanter of the femur. The second series $(24 \mathrm{~cm} \times 24 \mathrm{~cm}$ field of view; $7 \mathrm{~mm}$ slice thickness, $3 \mathrm{~mm}$ space between slices; 256 pixel $\times 256$ pixel matrix) traveled from the femoral head to the distal femur. Another series of proton-density images $(24 \mathrm{~cm} \times 24 \mathrm{~cm}$ field of view; $7 \mathrm{~mm}$ slice thickness; $3 \mathrm{~mm}$ space between slices; 256 pixel $\times 256$ pixel matrix) was collected with the hip flexed (approximately $60^{\circ}$ of flexion) and traveled from just below the iliac crest to just below the lesser trochanter of the femur. In all sets of images, the subject was relaxed with all muscles in the passive condition. The MR imaging protocol was approved by the Institutional Review Board of Stanford University.

We reconstructed the surface geometry of the muscles and bones from the MR images (Fig. 2). On each axialplane image, we manually outlined the boundaries of the muscles and bones of interest. The 3D polygonal surface models were generated for each structure from the set of 2D outlines (Nuages, INRIA, France).
We created solid hexahedral meshes of the muscles from the segmented surface models by using a mapping process in the finite-element mesh generator, TrueGrid (XYZ Scientific Applications, Livermore, CA). In this procedure, a "template mesh" that is in the shape of a cube (Fig. 3(A)) undergoes a mapping process (Fig. 3(B)) to create the "target mesh" (Fig. 3(C)) that represents the geometry of a specific muscle. The mapping $(\varphi)$ has a one-to-one correspondence so that each material coordinate in the template mesh $\left(\mathbf{X}^{\text {template }}\right)$ has a corresponding coordinate in the target mesh $\left(\mathbf{X}^{\text {target }}\right)$ :

$$
\mathbf{X}^{\text {target }}=\varphi\left(\mathbf{X}^{\text {template }}\right)
$$

The mapping function $(\varphi)$ was defined as follows. The template mesh has many "slices," each of which corresponds to an outline in the segmentation. The edges of each slice were projected on to the corresponding outline curve, and the internal nodes were smoothed to result in uniform element sizes within the slice.

For each muscle mesh, we identified the regions that corresponded to tendon (internal and/or external) and the regions that corresponded to origin and insertion areas, using a combination of the MR images, the reconstructed muscle and surface geometry, and knowledge of the anatomy. The origin of the psoas muscle was approximated as the area of the muscle in the most proximal image slice, therefore making the assumption (also made by others ${ }^{6,16,41}$ ) that the action of the psoas muscle in the lumbar region does not affect the action of the muscle at the hip joint.

\section{Specification of Fiber Geometries}

We developed a method to prescribe the geometry of fibers within the mesh for a variety of muscle architectures. Our method is based on defining a set of "template fiber geometries" which we then morph to create each muscle's "target fiber geometry." We created four template fiber geometries that were motivated by common classification schemes for muscle architecture (Fig. 4) 2,49 $^{\text {and describe }}$ the trajectory of many fibers within a regularly-shaped cube. The basis for each template fiber geometry is interpolation between multiple rational Bezier spline curves. We first describe our method for interpolating between spline curves and defining the analytical form for the fiber geometry.

Each fiber geometry template is created by linearly interpolating between at least two sets of control points in 3D space, $\mathbf{b}_{\mathbf{i}}^{\mathbf{a}}$ and $\mathbf{b}_{\mathbf{i}}^{\mathbf{b}}$, by the parameter, $s$, to define the control points for the intermediate spline, $\mathbf{b}_{\mathbf{i}}$ :

$$
\begin{aligned}
& \mathbf{b}_{\mathbf{i}}(s)=\mathbf{b}_{\mathbf{i}}^{\mathbf{a}}+\left(1-\frac{s}{\text { numfibs }}\right)\left(\mathbf{b}_{\mathbf{i}}^{\mathbf{b}}-\mathbf{b}_{\mathbf{i}}^{\mathbf{a}}\right) ; \\
& \quad 0 \leq s \leq \text { numfibs. } \\
& \mathbf{b}_{\mathbf{i}}(s)=\mathbf{b}_{\mathbf{i}}^{\mathbf{a}} ; \quad \text { numfibs }<s<\infty .
\end{aligned}
$$




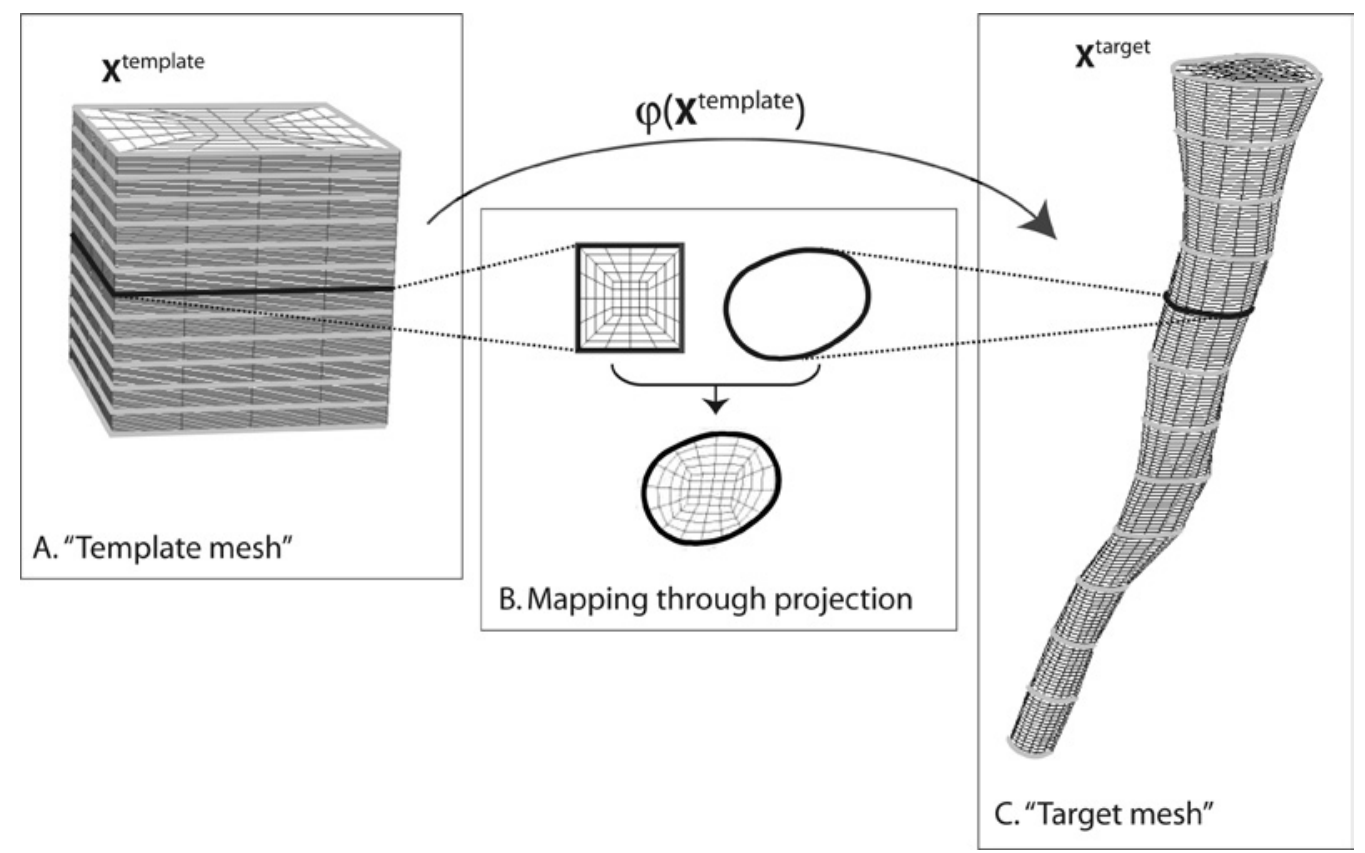

FIGURE 3. Creation of volumetric meshes of each muscle from segmented surface models. Hexahedral meshes were created by mapping a "template" hexahedral mesh (A) through a series of projections (B) to the "target" mesh (C). Each slice of the mesh corresponds to an outline of an anatomical structure created during segmentation (see Fig. 2).

We introduced weights for each control point so that the curvature of the splines varied continuously between the interpolated control points:

$$
w_{0}=1.0 ; \quad w_{1}(s)=s+1.0 ; \quad w_{2}=1.0 .
$$

The parameter, numfibs, defines the number of representative fibers in the region and is specified by the user. The expression for the template fiber geometry (fib $\left.{ }^{\text {template }}\right)$ is a function of $t$ (the fiber direction) and $s$ (transverse to the fiber direction):

$$
\mathbf{f i b}^{\text {template }}(t, s)=\frac{\sum_{i=0}^{2} w_{i}(s) \mathbf{b}_{\mathbf{i}}(s) B_{i}(t)}{\sum_{i=0}^{2} w_{i}(s) B_{i}(t)},
$$

where $B_{i}(t)$ are the quadratic Bernstein Basis Functions:

$$
B_{i}(t)=\frac{2}{(2-i) !}(1-t)^{2-i} t^{i} .
$$

The fiber direction (fibdir ${ }^{\text {template }}$ ) is the derivative of the fiber function along the parameter, $t$ :

$$
\operatorname{fibdir}^{\text {template }}(t, s)=\frac{\partial \mathbf{f i b}^{\text {template }}}{\partial t}(t, s) .
$$

We used this basic interpolation scheme to create a variety of template fiber geometries (Fig. 4), including parallel "simple" fibers, parallel "curved" fibers, "pennate" fibers, and "fanned" fibers. The "simple" fibers were a set of parallel lines traveling between opposite ends of the template mesh and consist of one set of control points, ${ }^{\mathrm{I}} \mathbf{b}_{\mathbf{i}}^{\mathbf{a}}$ and ${ }^{\mathrm{I}} \mathbf{b}_{\mathbf{i}}^{\mathbf{b}}$. The "pennate" fibers have attachments on either side of the template mesh and consist of two sets of control points, ${ }^{\mathrm{I}} \mathbf{b}_{\mathbf{i}}^{\mathbf{a}}$ and ${ }^{\mathrm{I}} \mathbf{b}_{\mathbf{i}}^{\mathbf{b}}$, and ${ }^{\mathrm{II}} \mathbf{b}_{\mathbf{i}}^{\mathbf{a}}$ and ${ }^{\mathrm{II}} \mathbf{b}_{\mathbf{i}}^{\mathbf{b}}$. The "curved" fibers have the attachments on the same side of the template cube and consist of two sets of control points, ${ }^{\mathrm{I}} \mathbf{b}_{\mathbf{i}}^{\mathbf{a}}$ and ${ }^{\mathrm{I}} \mathbf{b}_{\mathbf{i}}^{\mathbf{b}}$, and ${ }^{\mathrm{II}} \mathbf{b}_{\mathbf{i}}^{\mathbf{a}}$ and I $\mathbf{b}_{\mathbf{i}}^{\mathbf{b}}$. The "fanned" fibers have attachments on all four sides of the mesh and consist of four sets of control points, ${ }^{\mathrm{I}} \mathbf{b}_{\mathbf{i}}^{\mathbf{a}}$ and ${ }^{\mathrm{I}} \mathbf{b}_{\mathbf{i}}^{\mathbf{b}}$, ${ }^{\mathrm{II}} \mathbf{b}_{\mathbf{i}}^{\mathbf{a}}$ and ${ }^{\mathrm{I}} \mathbf{b}_{\mathbf{i}}^{\mathbf{b}}$, ${ }^{\mathrm{III}} \mathbf{b}_{\mathbf{i}}^{\mathbf{a}}$ and ${ }^{\mathrm{III}} \mathbf{b}_{\mathbf{i}}^{\mathbf{b}}$, and ${ }^{\mathrm{IV}} \mathbf{b}_{\mathbf{i}}^{\mathbf{a}}$ and ${ }^{\mathrm{IV}} \mathbf{b}_{\mathbf{i}}^{\mathbf{b}}$. While we present these four examples of fiber geometries, more complex fiber arrangements can be represented with this framework by adding more interpolating spline curves.

We sampled the template fiber function (fib) at various values of the parameters $t$ and $s$ to create representative template fibers (Fig. 4). The corresponding representative target fibers (Fig. 5) were then determined by applying the mapping function:

$$
\mathbf{f i b}^{\text {target }}=\varphi\left(\mathbf{f i b}^{\text {template }}\right) .
$$

We also used the fiber function to define the fiber direction for each element in the mesh, which served as the fiber direction input to the transversely-isotropic constitutive model (described later). For each integration point (i.e., point within an element for which the constitutive model is evaluated) in the finite-element mesh $\left(\mathbf{X}_{\mathrm{pnt}}^{\text {target }}\right)$, we first determined the coordinate in the template geometry by applying the inverse mapping:

$$
\mathbf{X}_{\mathrm{pnt}}^{\text {template }}=\varphi^{-1}\left(\mathbf{X}_{\mathrm{pnt}}^{\text {target }}\right) .
$$


A. "Simple"
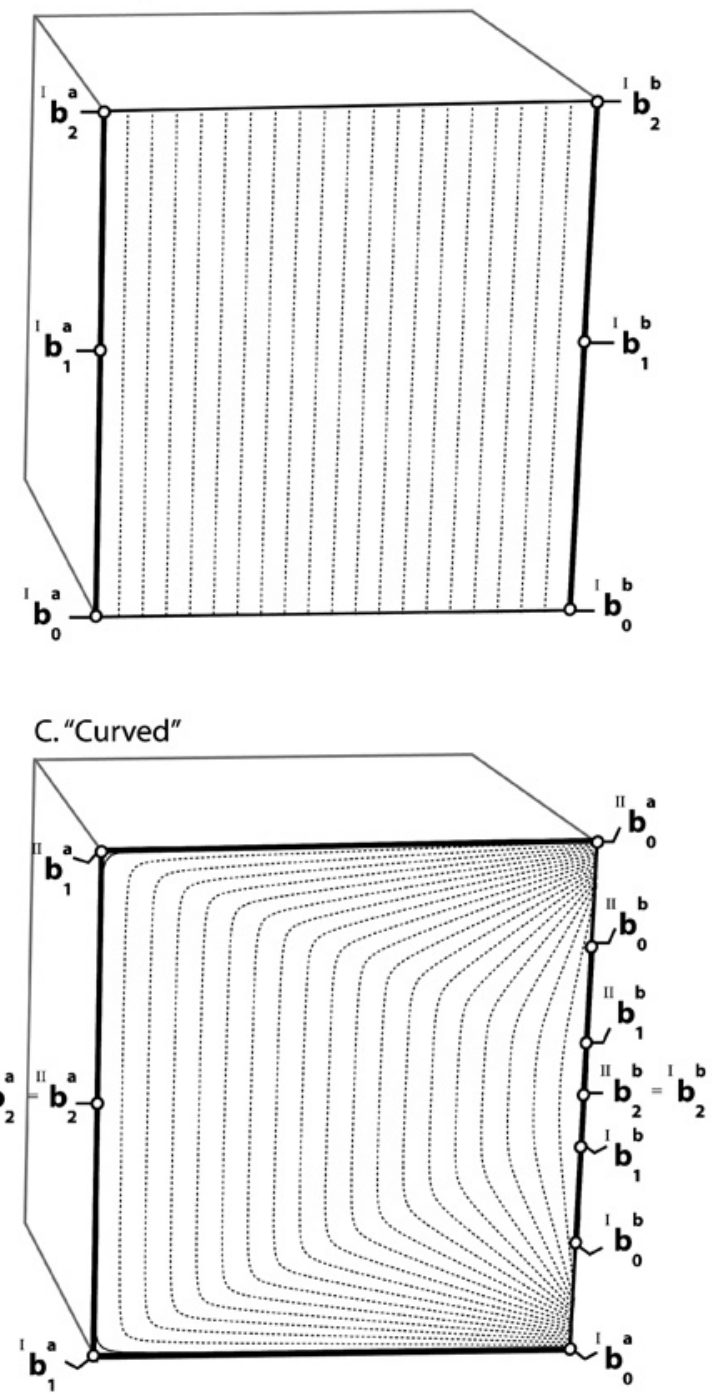

B. "Pennate"

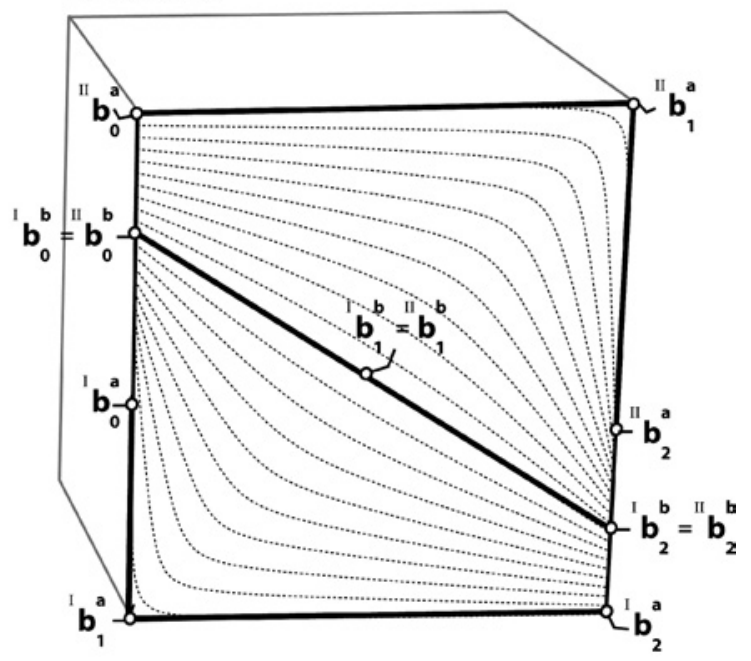

D. "Fanned"

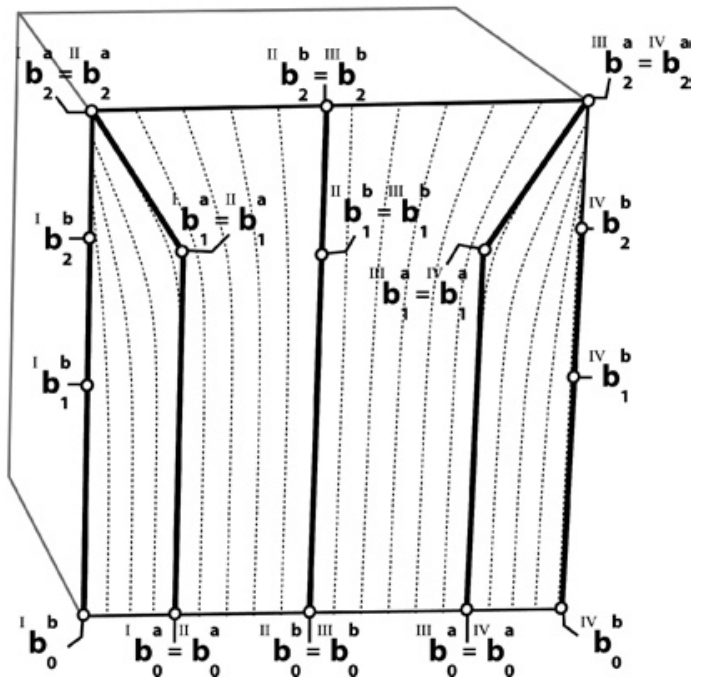

FIGURE 4. Fiber geometry templates used for parallel muscles (A), pennate muscles (B), curved muscles (C), and fanned muscles (D). The templates consist of interpolated rational Bezier spline curves. In the simplest case (A), only one set of spline curves are the basis for the interpolation. In the most complex case (D), four sets of spline curves are the basis for the interpolation.

We then used a least-squares method to determine corresponding coordinates $(t, s)$ in the fiber function, calculated the fiber direction at those coordinates (using Eq. (6)), and applied the mapping function to find the resulting fiber direction in the target geometry:

$$
\text { fibdir }^{\text {target }}=\varphi\left(\text { fibdir }^{\text {template }}\right) .
$$

\section{Constitutive Model}

We modeled muscle as a fiber-reinforced composite with transversely-isotropic material symmetry, similar to the approach previously used to represent ligament material behavior. ${ }^{46}$ The model uses an uncoupled form of the strain energy ${ }^{42,46}$ to simulate the nearly-incompressible behavior of muscle tissue. This uncoupled form additively separates the dilational and deviatoric responses of the tissue, giving rise to the following strain energy function $(\Psi)$ :

$$
\begin{aligned}
\Psi\left(B_{1}, B_{2}, \lambda, \alpha, J\right)= & W_{1}\left(B_{1}\right)+W_{2}\left(B_{2}\right) \\
& +W_{3}(\lambda, \alpha)+\frac{K}{2} \ln (J)^{2},
\end{aligned}
$$

where $\alpha$ is the activation level in the muscle, $K$ is the bulk modulus, and $B_{1}, B_{2}, \lambda$, and $J$ represent the along-fiber shear strain, cross-fiber shear strain, along-fiber stretch, and volume strain, respectively. The parameters for shear strains $\left(B_{1}\right.$ and $\left.B_{2}\right)$ were based on a new strain invariant set for transverse isotropy proposed by Criscione $e t$ al. ${ }^{13}$ The functional forms for $W_{1}$ and $W_{2}$ adopted for our model are 


\section{A. "Simple"}

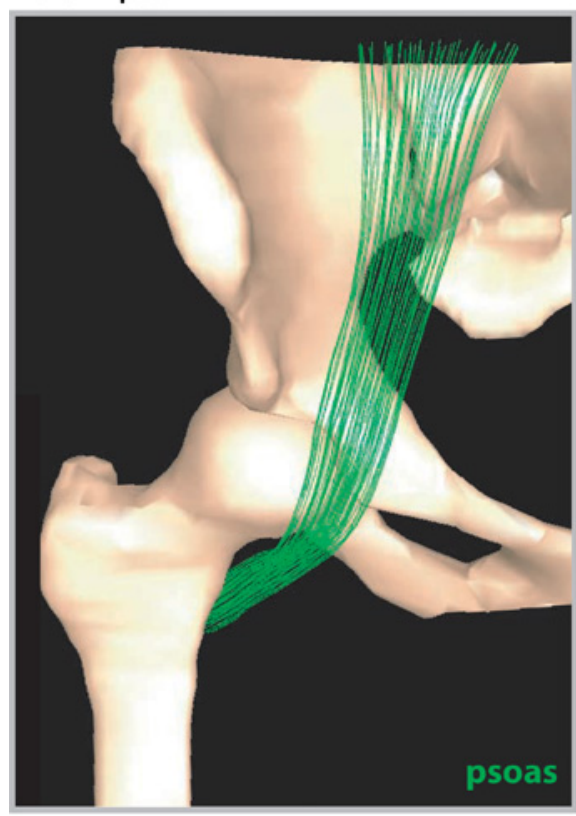

\section{C. “Curved”}

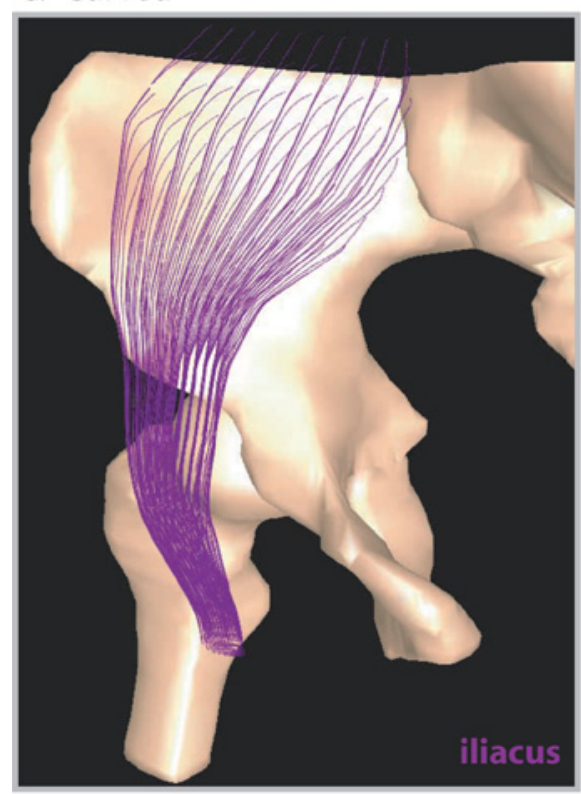

\section{B. “Pennate”}

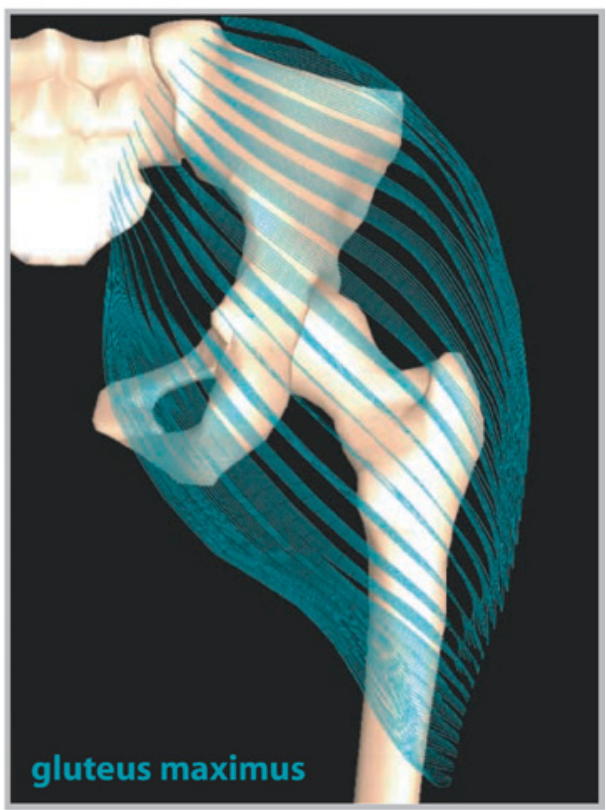

D. “Fanned"

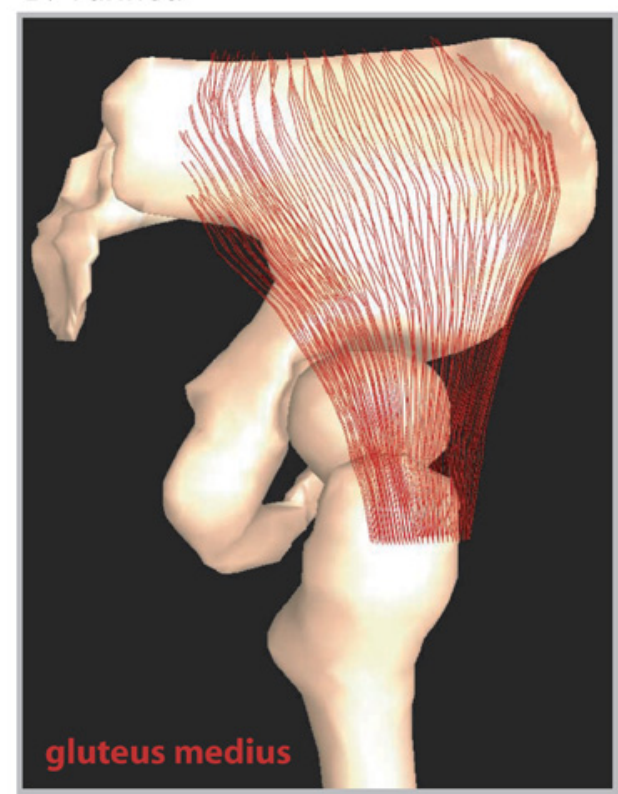

FIGURE 5. Examples of fiber geometries mapped to the psoas (A), gluteus maximus (B), iliacus (C), and gluteus medius (D) muscles.

as follows:

$$
W_{1}=G_{1} B_{1}^{2} \quad \text { and } \quad W_{2}=G_{2} B_{2}^{2},
$$

where $G_{1}$ and $G_{2}$ represent the effective along-fiber shear modulus and cross-fiber shear modulus, respectively. Equations (11) were used to represent both muscle and tendon, with different values for constants $G_{1}$ (5.0E2 Pa for muscle and 5.0E4 Pa for tendon) and $G_{2}$ (5.0E2 Pa for muscle and 5.0E4 $\mathrm{Pa}$ for tendon). The functional form for $W_{3}$ adopted for our model was as follows:

$$
\lambda \frac{\partial W_{3}^{\text {muscle }}}{\partial \lambda}=\sigma_{\max }\left(\alpha f_{\text {active }}^{\text {fiber }}(\lambda)+f_{\text {passive }}^{\text {fiber }}(\lambda)\right) \lambda / \lambda_{\text {off }},
$$

where $\sigma_{\max }$ is the maximum isometric stress, $\lambda_{\text {off }}$ is the fiber stretch at which the sarcomeres reach optimum length, and $f_{\text {active }}^{\text {fiber }}$ and $f_{\text {passive }}^{\text {fiber }}$ correspond to normalized active and passive (respectively) force-length relationships of a muscle fiber. ${ }^{49}$ The activation level $(\alpha)$ can be any value between 0 (no activation) and 1 (maximal activation). 
We also defined $W_{3}$ for tendinous tissue using a function that characterized the relationship between the Cauchy stress in the tendon $\left(\sigma^{\text {tendon }}\right)$ and the fiber stretch $(\lambda)$ :

$$
\lambda \frac{\partial W_{3}^{\text {tendon }}}{\partial \lambda}=\sigma^{\text {tendon }}(\lambda)
$$

The expression for $\sigma^{\text {tendon }}$ and the associated material constants were defined to be consistent with the published stress-strain relationship for tendon. ${ }^{49}$ Further description of the constitutive model can be found in Blemker et al. ${ }^{10}$

\section{Simulation Procedure}

Coordinate systems were specified for the pelvis and femur based on anatomical landmarks, similar to Arnold et al. ${ }^{6}$ The medial-lateral axis of the pelvis was defined by the vector from the right anterior superior iliac spine (ASIS) to the left ASIS. The frontal plane was defined by the ASIS vector and a point on the pubic tubercle. The hip joint was represented as a ball-and-socket joint, and the center of the hip was determined by fitting a sphere to the surface of the femoral head. The nodes in the origin and insertion areas of the muscles were specified to attach rigidly to their corresponding bone (pelvis or femur). As a result, the displacement boundary conditions for each muscle mesh were defined by specifying a set of hip joint angles. The bones were considered as rigid bodies, and a no-friction penalty contact formulation ${ }^{24}$ was used to prevent penetration between muscles and bones and between neighboring muscles. The simulations were performed in NIKE3D, ${ }^{39}$ a nonlinear implicit finite-element solver. The solutions were quasi-static and solved incrementally. Simulation times were on the order of 5-10 CPU hours on a Silicon Graphics Origin 3800 shared memory supercomputer (Silicon Graphics, Mountain View, CA). The results of each simulation were imported into a graphics-based musculoskeletal modeling environment. ${ }^{15}$

\section{Evaluations of the Models}

We first evaluated the models by comparing the muscle fiber moment arms for hip flexion-extension, internalexternal rotation, and abduction-adduction with published experiments and/or models. To do this, we performed simulations in which we specified a range of hip flexion, adduction, or internal rotation angles. We then tracked selected fibers within the finite-element solution to obtain fiber lengths $\left(l^{\text {fiber }}\right)$ as a function of joint angle $(\theta)$ and fit a fourth-order polynomial to each function, $l^{\text {fiber }}(\theta)$. Individual fiber moment arms ( $\left.m a^{\text {fiber }}\right)$ were calculated according to the principal of virtual work, ${ }^{3} m a^{\text {fiber }}=\partial l^{\text {fiber }} / \partial \theta$. We compared the fiber moment arms predicted by the models with moment arms determined from anatomical measurements ${ }^{6,14,20,36}$ and published models of the lower extremity (with series-of-line-segment representations of muscle). ${ }^{6,16}$
We also evaluated the models by comparing the changes in shape of the 3D muscle models with changes in shape measured from the MR images we acquired with the hip extended and flexed positions. We used an iterative closestpoint algorithm ${ }^{9}$ to register the surface reconstructions of the pelvis and femur in the extended position to the pelvis and femur surfaces in the flexed position. Based on these registrations, we calculated the relative position of the femur with respect to the pelvis, which corresponded to $55^{\circ}$ of hip flexion, $6^{\circ}$ of hip abduction, and $7^{\circ}$ of external rotation (rotations defined in that order). We applied this hip position to the finite-element model (through 50 increments), which calculated the muscle shape changes resulting from the change in hip position. To estimate the distance between the model surfaces and the surfaces segmented from the MR images, we projected each point of the segmented surface onto the surface of the 3D muscle model. These projections gave us a distance error for each point on the segmented surface; we then calculated the average and root mean square (RMS) errors across all the points. Since the segmented surface points were evenly distributed across the segmented surface, the resulting error measures were not highly sensitive to the number of points used.

\section{RESULTS}

The peak hip flexion moment arms for the psoas fibers (Fig. 6(C)) ranged from 2.0 to $3.0 \mathrm{~cm}$ and for the iliacus fibers (Fig. 6(D)) ranged from 2.5 to $5.2 \mathrm{~cm}$. The larger variation in peak hip flexion moment arms across iliacus fibers reflects the fact that the iliacus has a broad origin on the pelvic fossa. The average of the psoas fiber hip flexion moment arms were similar to hip flexion moment arms determined experimentally ${ }^{6}$ and the largest of iliacus fiber hip flexion moment arms were similar to moment arms estimated from a model of the lower extremity that used a series of line segments. ${ }^{16}$ The peak hip adduction moment arms for the psoas fibers (Fig. $6(\mathrm{G})$ ) ranged from 1.5 to $2.0 \mathrm{~cm}$ and for the iliacus fibers (Fig. $6(\mathrm{H})$ ) ranged from 0.0 to $1.5 \mathrm{~cm}$. The peak hip internal rotation moment arms for the psoas fibers (Fig. $6(\mathrm{~K})$ ) ranged from 0.2 to $0.8 \mathrm{~cm}$ and for the iliacus fibers (Fig. 6(L)) ranged from 0.2 to $0.9 \mathrm{~cm}$.

The peak hip extension moment arms for the gluteus maximus fibers ranged from 1.0 to $7.0 \mathrm{~cm}$ (Fig. 7(C)). Previous experiments, ${ }^{20}$ which represented gluteus maximus with a single line of action, had a single peak moment arm that was slightly larger than the largest 3D model fiber moment arm. The peak hip extension moment arms for the gluteus medius fibers (Fig. 7(D)) ranged from -2.0 to $2.0 \mathrm{~cm}$. For both of the gluteal muscles, the hip flexion moment arms determined experimentally ${ }^{20}$ varied more with hip flexion than the moment arms computed with the 3D models. The peak hip adduction moment arms for the gluteus maximus fibers (Fig. 7(G)) ranged 

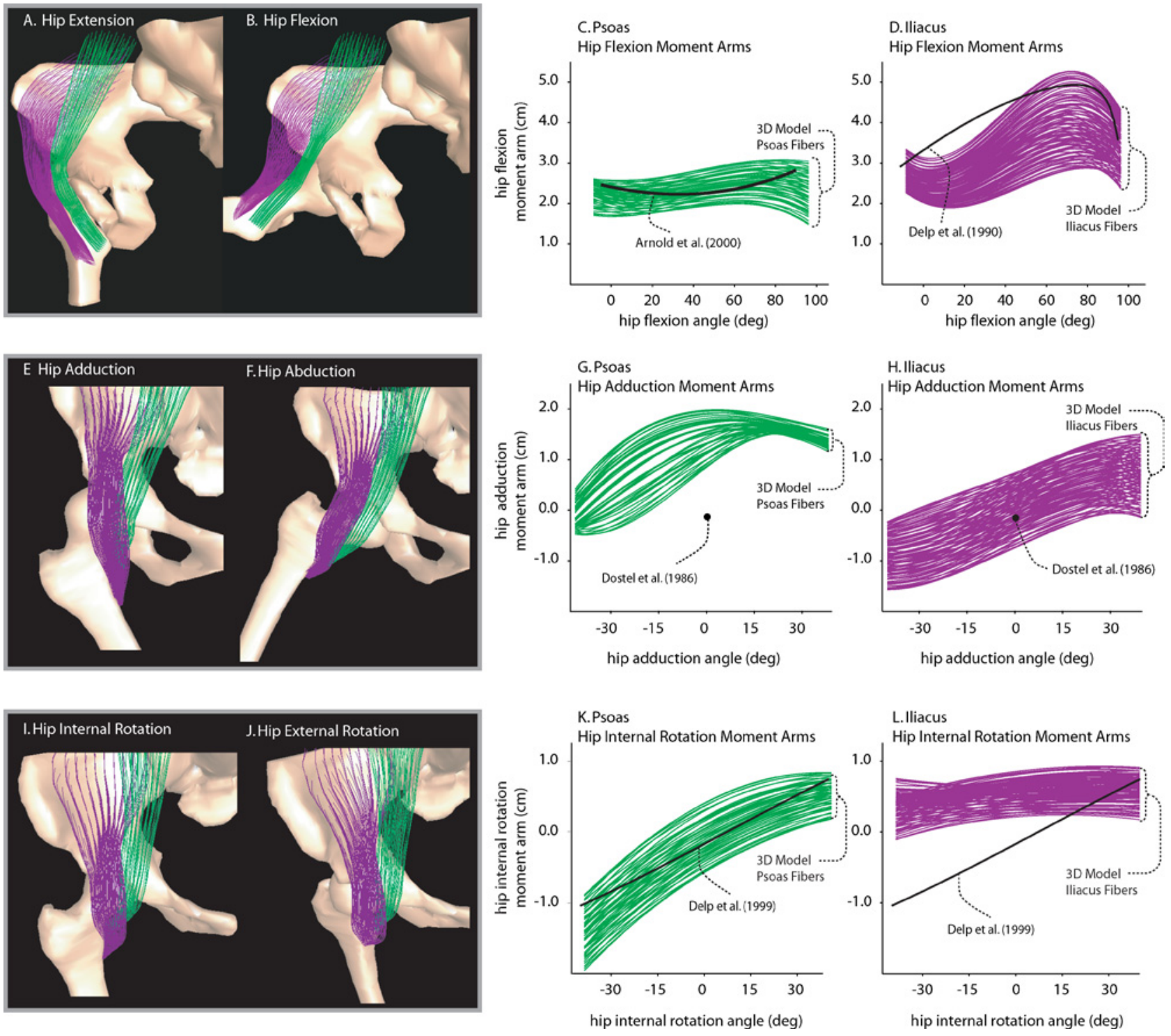

FIGURE 6. The psoas (green) and iliacus (purple) muscle models with the hip extended/flexed (A/B), adducted/abducted (E/F), internally/externally rotated $(\mathrm{I} / \mathrm{J})$. Hip flexion $(\mathrm{C}, \mathrm{D})$, adduction $(\mathrm{G}, \mathrm{H})$, and internal rotation $(\mathrm{K}, \mathrm{L})$ moment arms predicted by the models are compared to previously reported data. Psoas hip flexion moment arms (C) are compared to experimental measurements, ${ }^{6}$ and the iliacus hip flexion moment arms (D) are compared to a series-of-line-segments model. ${ }^{16}$ Psoas (G) and iliacus (H) hip adduction moment arms are compared to experimental measurements in the neutral position, which considered the two muscles as one unit. $^{20}$ Psoas (K) and iliacus (L) hip internal rotation moment arms are compared to a previously-published model which was validated by experimental measurements and also considered the two muscles as one unit. ${ }^{14}$

from 0.1 to $7.0 \mathrm{~cm}$, and for the gluteus medius fibers (Fig. $7(\mathrm{H}))$ ranged from -3.0 to $1.5 \mathrm{~cm}$. The peak hip internal rotation moment arms for the gluteus maximus fibers (Fig. $7(\mathrm{~K})$ ) ranged from -3.5 to $-0.2 \mathrm{~cm}$, and for the gluteus medius fibers (Fig. 7(L)) ranged from -2.3 to $2.1 \mathrm{~cm}$.

There was generally good agreement between the shape changes predicted by the models and the MRI data (Fig. 8 , Table 1). The average error between the psoas surfaces was $1.7 \mathrm{~mm}$, and the average error between the iliacus surfaces was $2.0 \mathrm{~mm}$. The average error between the gluteus maximus surfaces was $5.2 \mathrm{~mm}$, and the average error between the gluteus medius surfaces was $2.3 \mathrm{~mm}$. As a comparison, the average error between the registered pelvis surfaces was $1.3 \mathrm{~mm}$, and the average error between the registered femur surfaces was $1.2 \mathrm{~mm}$. The larger error between the gluteus maximus surfaces was possibly due to differences in boundary conditions between the images and the models. The posterior surface of the muscle was in contact with the table of the scanner during image acquisition; this external force was not included in the model. 

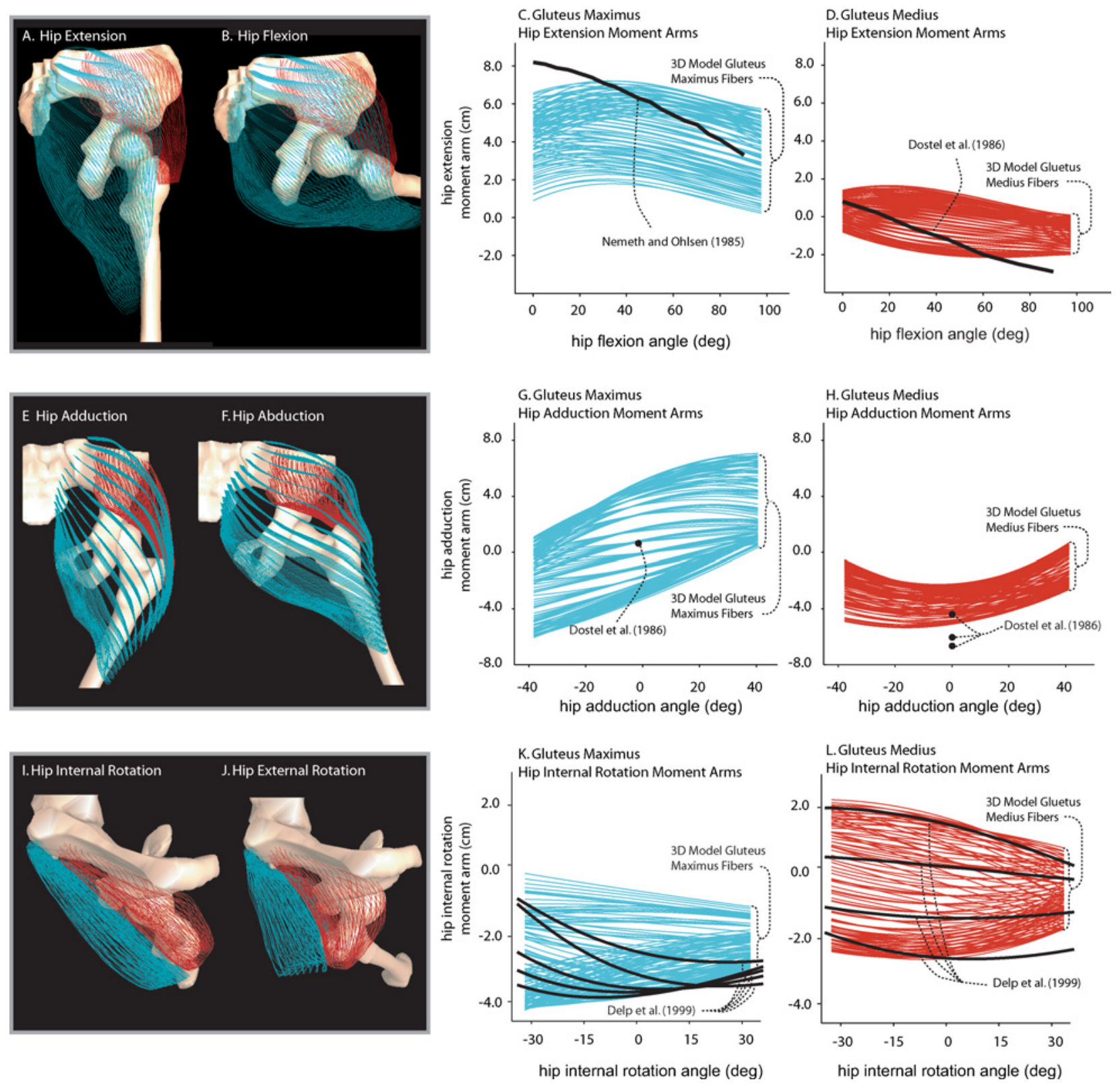

FIGURE 7. The gluteus maximus (cyan) and gluteus medius (red) muscle models with the hip extended/flexed (A/B), adducted/abducted (E/F), internally/externally rotated (I/J). Hip extension (C,D), adduction (G,H), and internal rotation (K,L) moment arms predicted by the models are compared to previously reported data. Gluteus maximus (C) and medius (D) hip extension moment arms are compared to experimental measurements. ${ }^{20,37}$ Gluteus maximus (G) and medius (H) hip adduction moment arms are compared to experimental measurements in the neutral position. ${ }^{20}$ Gluteus maximus (K) and medius (L) hip internal rotation moment arms are compared to a previously-published model which separated the muscles into multiple compartments and was validated by experimental measurements. ${ }^{14}$

\section{DISCUSSION}

This new 3D formulation for representing muscle can characterize muscles with complex geometry and represent the variation in moment arms among fibers in a muscle. In contrast to line-segments path representations, 3D models can represent complex path motion through multiple degrees of freedom without the need for defining via points or wrapping surfaces. The predictions of change in shapes for the 3D models compared well with shape changes estimated from static MR image data in two positions. We found that fiber moment arms have a considerable variation within each muscle, indicating that the common assumption that all fiber lengths and excursions within muscle are the same may not be valid, at least for the muscles examined here. 


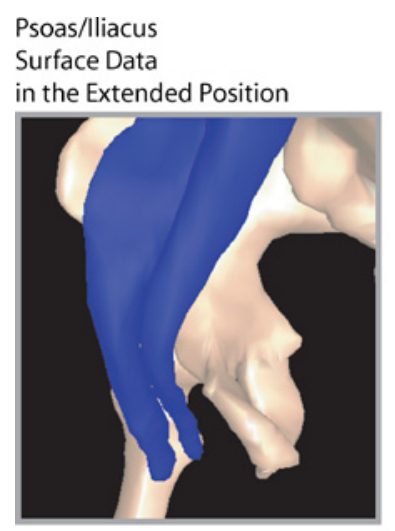

\begin{abstract}
Psoas/liacus
Surface Data

in the Flexed Position
\end{abstract}
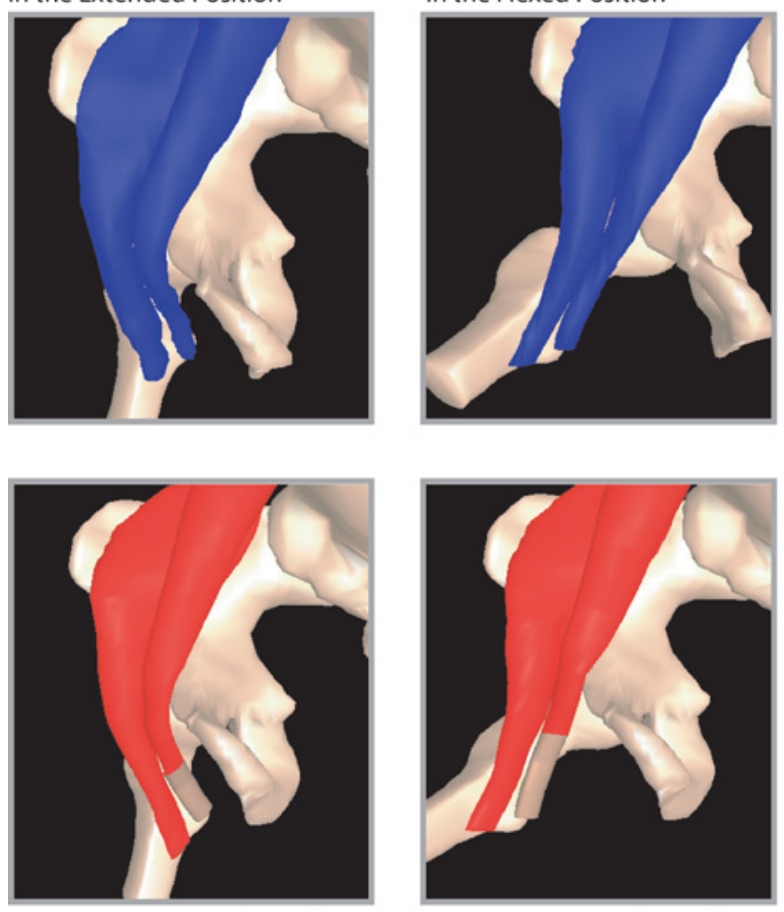

Psoas/liacus

3D Model

in the Extended Position

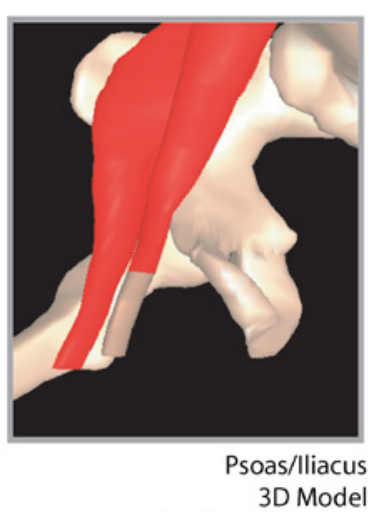

in the Flexed Position
Gluteus Maximus/Medius

Surface Data

in the Extended Position
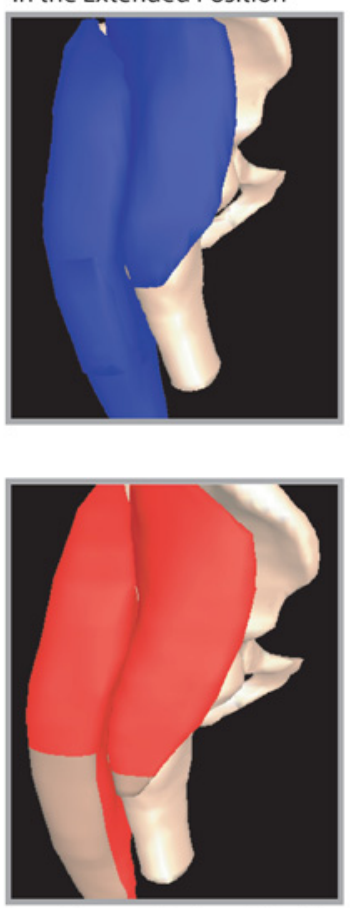

Gluteus Maximus/Medius

3D Model

in the Extended Position
Gluteus Maximus/Medius

Surface Data

in the Flexed Position
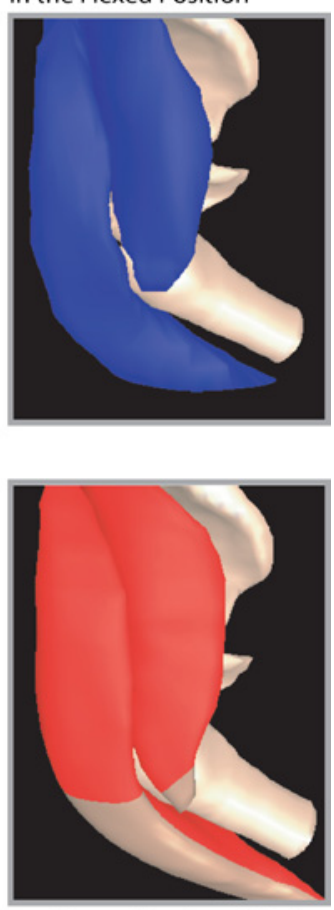

Gluteus Maximus/Medius 3D Model

in the Flexed Position

FIGURE 8. Comparison of segmented MR surface data acquired from the hip in two positions (top row) with changes in shape predicted by the 3D models (bottom row). The two different shades/colors in the 3D models distinguish between muscle tissue elements (darker/red) and tendon tissue elements (lighter/taupe).

Previous studies have described continuum representations of muscle ${ }^{23,30,31,43}$ and used them, for example, to investigate intramuscular pressure ${ }^{28}$ and understand myofascial force transmission. ${ }^{48}$ These models represent the complex nonlinear behavior of muscle tissue; however, they have not incorporated realistic geometric arrangements of muscle fibers or muscle-bone and muscle-muscle surface contact. Agur et al. ${ }^{1}$ developed methods to capture and model the 3D arrangement of muscle fibers from anatomical dissection, which can provide insight into the 3D complexity of muscle architecture; however, these models do not predict the changes in shape or fiber geometry as joints move. The formulation presented here advances these methods by combining 3D representations of fiber arrangements with representations of the nonlinear constitutive behavior of muscle and representations of muscle-bone and musclemuscle interactions to predict muscle shape, lengths, and moment arms through a range of joint positions.

We found that representation of muscles as 3D bodies that interact with other muscles and underlying structures resulted in different variations in moment arms with joint angles than predicted by previous experimental measurements and series-of-line-segments models. For example, the gluteus maximus and medius muscle moment arms predicted by the 3D models varied less with hip flexion than predicted by experiments. In our models, the path motion was constrained by interactions between the entire boundary of the muscles and the underlying bones. Furthermore,

TABLE 1. Distances between muscle surfaces.

\begin{tabular}{lcccc}
\hline & $\begin{array}{c}\text { Maximum } \\
\text { error }(\mathrm{mm})\end{array}$ & $\begin{array}{c}\text { Average } \\
\text { error }(\mathrm{mm})\end{array}$ & $\begin{array}{c}\text { Root-mean squared } \\
\text { error }(\mathrm{mm})\end{array}$ & $\begin{array}{c}\text { Number of } \\
\text { points }\end{array}$ \\
\hline Psoas & 8.1 & 1.7 & 2.2 & 791 \\
lliacus & 7.0 & 2.0 & 2.5 & 795 \\
Gluteus maximus & 25.1 & 5.2 & 7.5 & 845 \\
Gluteus medius & 9.4 & 2.3 & 3.1 & 394 \\
\hline
\end{tabular}


because the muscle is considered as a 3D continuum, these external constraints also restrict the movement of the rest of the muscle tissue, based on the transverse mechanical properties and the volume preservation constraint. Experimental measurements of moment arms generally require removal of some surrounding structures, and line-segment representations do not generally represent the interactions between muscles. Moreover, because experiments and previous models only represent the muscle using a line or wire, the resulting internal constraints would not be imposed. As a result, the muscles in previous experiments and models are, in some cases, less constrained than they are in vivo. In contrast, the mechanics-based approach presented in this paper explicitly represents the constraints that external and internal structures place on each muscle.

This mechanics-based approach for representing muscle could also be applied to characterizing the effects of muscle activation on muscle geometry. As muscles develop force, they bulge and interact with each other and other structures. Models have generally assumed that muscle moment arms are independent of muscle force. The models presented here can be used to test this assumption by applying various muscle activation levels to the constitutive model. The effects of muscle activation will likely depend on the muscle's architecture and geometry as well as the surrounding structures.

The 3D muscle models have more input parameters than the line-segments path models. While some of the material properties have been measured (e.g., force-length behavior of a fiber), data on which to base other parameters have not been determined experimentally (e.g., the resistance to along-fiber and cross-fiber shear). Data exist for the average optimal fiber length for each of the lower limb muscles; ${ }^{21,47}$ however, no comprehensive data exist that characterize the variation in optimal fiber lengths within the lower-limb muscles. Detailed architecture measurements that capture the fiber trajectories in the muscle and the distribution of optimal fiber lengths are needed. Though more data is needed to better define the geometry and material properties of 3D models, the parameters of the models we introduced have an anatomical and physical basis and can be measured in future experiments.

The 3D muscle models also require new types of data for validation. We compared the moment arms estimated by the $3 \mathrm{D}$ models with moment arms determined using traditional techniques, like the tendon excursion method. ${ }^{3}$ These measurements fall short of fully testing the models. For each joint position, the measurements provide only one value for the moment arm of a muscle compartment, making the same assumption that all fibers within each muscle compartment have the same moment arm. An alternative could be to use the dynamic ultrasound measurements of changes in fascicle geometry during joint motion ${ }^{34}$ to test the accuracy of fiber lengths and moment arms predicted by the 3D muscle models. The second evaluation we made in this paper was to compare the muscle shape changes with static MR images in multiple joint positions. While we show a preliminary comparison between two joint positions, one could imagine a more comprehensive comparison over a range of joint angles in a larger number of subjects. Deformations from these models could also be evaluated with dynamic MR imaging, such as cine phase-contrast $\mathrm{MR}^{8}$ or real-time MR, ${ }^{7}$ which give muscle tissue velocities and displacements throughout a motion cycle.

Currently, it is impractical to use this 3D muscle representation in all situations for which musculoskeletal models are used. For example, the computational expense of these models makes them incompatible with simulations that are already computationally expensive, like controlling a forward dynamic simulation of movement. ${ }^{4}$ However, the results from the 3D models could be used in a dynamic simulation by fitting regression equations to the average muscle lengths and moment arms ${ }^{33}$ predicted by the models.

Models of musculoskeletal system are used in a broad range of scientific studies. Models of musculoskeletal geometry are used to simulate orthopedic procedures, such as osteotomies, ${ }^{11,40}$ tendon transfers, ${ }^{17,25,32,35}$ and tendon lengthenings. ${ }^{18,19}$ Musculoskeletal models, combined with dynamic simulation, are used to study normal ${ }^{5}$ and pathological human movement. ${ }^{38}$ The conclusions from these studies depend on accurate representation of muscle architecture and geometry. The methods introduced here offer the potential to improve our ability to represent complex muscle geometry and architecture and enhance the accuracy of models of the musculoskeletal system for a wide variety of applications.

\section{ACKNOWLEDGMENTS}

We are thankful to Garry Gold, Mario Davinelli, Jeff Weiss, Deanna Asakawa, Peter Pinsky, Joseph Teran, Victor Ng-Thow-Hing, Ron Fedkiw, the Lawrence Livermore National Labs, and the Stanford Bio-X supercomputer facility. Funding for this work was provided by the National Institutes of Health Grants HD38962 and HD33929, a graduate fellowship from the National Science Foundation, and a Stanford Bio-X IIP Grant.

\section{REFERENCES}

${ }^{1}$ Agur, A. M., V. Ng-Thow-Hing, K. A. Ball, E. Fiume, and N. H. McKee. Documentation and three-dimensional modelling of human soleus muscle architecture. Clin. Anat. 16(4):285-293, 2003.

${ }^{2}$ Alexander, R. M., and R. F. Ker. The architecture of leg muscles. In: Multiple Muscle Systems, edited by J. M. Winters and S. L. Woo. New York: Springer-Verlag, 1990, p. 568-577.

${ }^{3}$ An, K. N., K. Takahashi, T. P. Harrigan, and E. Y. Chao. Determination of muscle orientations and moment arms. J. Biomech. Eng. 106(3):280-282, 1984.

${ }^{4}$ Anderson, F. C., and M. G. Pandy. Dynamic optimization of human walking. J. Biomech. Eng. 123(5):381-390, 2001. 
${ }^{5}$ Anderson, F. C., and M. G. Pandy. Individual muscle contributions to support in normal walking. Gait Posture 17(2):159-169, 2003.

${ }^{6}$ Arnold, A. S., S. Salinas, D. J. Asakawa, and S. L. Delp. Accuracy of muscle moment arms estimated from MRI-based musculoskeletal models of the lower extremity. Comput. Aided Surg. 5(2):108-119, 2000.

${ }^{7}$ Asakawa, D. S., K. S. Nayak, S. S. Blemker, S. L. Delp, J. M. Pauly, D. G. Nishimura, and G. E. Gold. Real-time imaging of skeletal muscle velocity. J. Magn. Reson. Imaging. 18(6):734739, 2003.

${ }^{8}$ Asakawa, D. S., G. P. Pappas, S. S. Blemker, J. E. Drace, and S. L. Delp. Cine phase-contrast magnetic resonance imaging as a tool for quantification of skeletal muscle motion. Semin. Musculoskelet. Radiol. 7(4):287-295, 2003.

${ }^{9}$ Besl, P. J., and N. D. McKay. A method for registration of 3-D shapes. IEEE Trans. Pattern Anal. Machine Intell. 14(2):239256, 1992.

${ }^{10}$ Blemker, S. S., P. M. Pinsky, and S. L. Delp. A 3D Model of muscle reveals the causes of nonuniform strains in the biceps brachii. J. Biomech. 38(4):657-665, 2005.

${ }^{11}$ Brand, R. A., and D. R. Pedersen. Computer modeling of surgery and a consideration of the mechanical effects of proximal femoral osteotomies. In: The Hip: Proceedings of the 12th Open Scientific Meeting of the Hip Society, 1984. St. Louis: C. V. Mosby.

${ }^{12}$ Chao, E. Y.S., J. D. Lynch, and M. J. Vanderploeg. Simulation and animation of musculoskeletal joint system. J. Biomech. Eng. 115:562-568, 1993.

${ }^{13}$ Criscione, J. C., A. S. Douglas, and W. C. Hunter. Physically based strain invariant set for materials exhibiting transversely isotropic behavior. J. Mech. Phys. Sol. 49:871-897, 2001.

${ }^{14}$ Delp, S. L., W. E. Hess, D. S. Hungerford, and L. C. Jones. Variation of rotation moment arms with hip flexion. J. Biomech. 32(5):493-501, 1999.

${ }^{15}$ Delp, S. L., and J. P. Loan. A computational framework for simulation and analysis of human and animal movement. IEEE Comput. Sci. Eng. 2(5):46-55, 2000.

${ }^{16}$ Delp, S. L., J. P. Loan, M. G. Hoy, F. E. Zajac, E. L. Topp, and J. M. Rosen. An interactive graphics-based model of the lower extremity to study orthopaedic surgical procedures. IEEE Trans. Biomed. Eng. 37(8):757-767, 1990.

${ }^{17}$ Delp, S. L., D. A. Ringwelski, and N. C. Carroll. Transfer of the rectus femoris: Effects of transfer site on moment arms about the knee and hip. J. Biomech. 27(10):1201-1211, 1994.

${ }^{18}$ Delp, S. L., K. Statler, and N. C. Carroll. Preserving plantar flexion strength after surgical treatment for contracture of the triceps surae: A computer simulation study. J. Orthop. Res. 13(1):96104, 1995.

${ }^{19}$ Delp, S. L., and F. E. Zajac. Force- and moment-generating capacity of lower-extremity muscles before and after tendon lengthening. Clin Orthop. (284):247-259, 1992.

${ }^{20}$ Dostal, W. F., G. L. Soderberg, and J. G. Andrews. Actions of hip muscles. Phys. Ther. 66(3):351-361, 1986.

${ }^{21}$ Friederich, J. A., and R. A. Brand. Muscle fiber architecture in the human lower limb. J. Biomech. 23(1):91-95, 1990.

${ }^{22}$ Garner, B. A., and M. G. Pandy. The Obstacle-Set Method for Representing Muscle Paths in Musculoskeletal Models. Comput. Methods Biomech. Biomed. Eng. 3(1):1-30, 2000.

${ }^{23}$ Gielen, A. W., C. W. Oomens, P. H. Bovendeerd, T. Arts, and J. D. Janssen. A finite element approach for skeletal muscle using a distributed moment model of contraction. Comput. Methods Biomech. Biomed. Eng. 3(3):231-244, 2000.

${ }^{24}$ Hallquist, J. O., G. L. Goudreau, and D. J. Bension. Sliding interfaces with contact-impact in large-scale Lagrangian computations. Int. J. Numer. Methods Eng. 51:107-137, 1985.
${ }^{25}$ Herrmann, A. M., and S. L. Delp. Moment arm and forcegenerating capacity of the extensor carpi ulnaris after transfer to the extensor carpi radialis brevis. J. Hand. Surg. [Am]. 24(5):1083-1090, 1999.

${ }^{26}$ Herzog, W., and H. E. ter Keurs. Force-length relation of in-vivo human rectus femoris muscles. Pflugers Arch. 411(6):642-647, 1988.

${ }^{27}$ Hoy, M. G., F. E. Zajac, and M. E. Gordon. A musculoskeletal model of the human lower extremity: The effect of muscle, tendon, and moment arm on the moment-angle relationship of musculotendon actuators at the hip, knee, and ankle. J. Biomech. 23(2):157-169, 1990.

${ }^{28}$ Jenkyn, T. R., B. Koopman, P. Huijing, R. L. Lieber, and K. R. Kaufman. Finite element model of intramuscular pressure during isometric contraction of skeletal muscle. Phys. Med. Biol. 47(22):4043-4061, 2002.

${ }^{29}$ Jensen, R. H., and D. T. Davy. An investigation of muscle lines of action about the hip: A centroid line approach vs. the straight line approach. J. Biomech. 8:103-110, 1975.

${ }^{30}$ Johansson, T., P. Meier, and R. Blickhan. A finite-element model for the mechanical analysis of skeletal muscles. J. Theor. Biol. 206(1):131-149, 2000.

${ }^{31}$ Kojic, M., S. Mijailovic, and N. Zdravkovic. Modelling of muscle behaviour by the finite element method using Hill's threeelement model. Int. J. Numer. Method Eng. 43:941-953, 1998.

${ }^{32}$ Lieber, R. L., and J. Friden. Intraoperative measurement and biomechanical modeling of the flexor carpi ulnaris-toextensor carpi radialis longus tendon transfer. J. Biomech. Eng. 119(4):386-391, 1997.

${ }^{33}$ Menegaldo, L. L., A. T. Fleury, and H. I. Weber. Moment arms and musculotendon lengths estimation for a three-dimensional lower-limb model. J. Biomech. 37(9):1447-1453, 2004.

${ }^{34}$ Muramatsu, T., T. Muraoka, Y. Kawakami, A. Shibayama, and T. Fukunaga. In vivo determination of fascicle curvature in contracting human skeletal muscles. J. Appl. Physiol. 92(1):129134, 2002.

${ }^{35}$ Murray, W. M., A. M. Bryden, K. L. Kilgore, and M. W. Keith. The influence of elbow position on the range of motion of the wrist following transfer of the brachioradialis to the extensor carpi radialis brevis tendon. J. Bone Joint Surg. Am. 84A(12):2203-2210, 2002.

${ }^{36}$ Nemeth, G., and H. Ohlsen. In vivo moment arm lengths for hip extensor muscles at different angles of hip flexion. J. Biomech. 18(2):129-140, 1985.

${ }^{37}$ Nemeth, G., and H. Ohlsen. Moment arm lengths of trunk muscles to the lumbosacral joint obtained in vivo with computed tomography. Spine 11(2):158-160, 1986.

${ }^{38}$ Piazza, S. J., and S. L. Delp. The influence of muscles on knee flexion during the swing phase of gait. J. Biomech. 29(6):723733, 1996.

${ }^{39}$ Puso, M. A., B. N. Maker, R. M. Ferencz, and J. O. Hallquist. Nike3d: A Nonlinear, Implicit, Three-Dimensional Finite Element Code for Solid and Structural Mechanics, 2002. Lawrence Livermore National Lab Technical Report.

${ }^{40}$ Schmidt, D. J., A. S. Arnold, N. C. Carroll, and S. L. Delp. Length changes of the hamstrings and adductors resulting from derotational osteotomies of the femur. J. Orthop. Res. 17(2):279-285, 1999.

${ }^{41}$ Schutte, L. M., S. W. Hayden, and J. R. Gage. Lengths of hamstrings and psoas muscles during crouch gait: Effects of femoral anteversion. J. Orthop. Res. 15(4):615-621, 1997.

${ }^{42}$ Simo, J. C., and R. L. Taylor. Quasi-incompressible finite elasticity in principal stretches: Continuum basis and numerical examples. Comp. Method Appl. Mech. Eng. 51:273-310, 1991.

${ }^{43}$ Teran, J., S. S. Blemker, V. Ng-Thow Hing, and R. Fedkiw. Finite-volume method for simulation of muscle tissue. In: $A C M$ 
SIGGRAPH/Eurographics Symposium on Computer Animation (SCA), edited by D.B.A.M. Lin, 2003, p. 68-74.

${ }^{44}$ Van der Helm, F. C., and R. Veenbaas. Modelling the mechanical effect of muscles with large attachment sites: Application to the shoulder mechanism. J. Biomech. 24(12):1151-1163, 1991.

${ }^{45}$ Van der Helm, F. C., H. E. Veeger, G. M. Pronk, L. H. Van der Woude, and R. H. Rozendal. Geometry parameters for musculoskeletal modelling of the shoulder system. J. Biomech. 25(2):129-144, 1992.

${ }^{46}$ Weiss, J. A., B. N. Maker, and S. Govindjee. Finite element implementation of incompressible, transversely isotropic hy- perelasticity. Comp. Method Appl. Mech. Eng. 135:107-128, 1996.

${ }^{47}$ Wickiewicz, T. L., R. R. Roy, P. L. Powell, and V. R. Edgerton. Muscle architecture of the human lower limb. Clin. Orthop. Relat. Res. 179:275-283, 1983.

${ }^{48}$ Yucesoy, C. A., B. H. Koopman, P. A. Huijing, and H. J. Grootenboer. Three-dimensional finite element modeling of skeletal muscle using a two-domain approach: Linked fiber-matrix mesh model. J. Biomech. 35(9):1253-1262, 2002.

${ }^{49}$ Zajac, F. E. Muscle and tendon: Properties, models, scaling, and application to biomechanics and motor control. Crit. Rev. Biomed. Eng. 17:359-411, 1989. 